Corrigendum

\title{
Corrigendum to "Combined replacement of fishmeal and fish oil in European sea bass (Dicentrarchus labrax): Production performance, tissue composition and liver morphology" [Aquaculture 474 (2017) 101-112]
}

S. Torrecillas ${ }^{\mathrm{a}, *}$, L. Robaina ${ }^{\mathrm{a}}$, M.J. Caballero ${ }^{\mathrm{a}}$, D. Montero ${ }^{\mathrm{a}}$, G. Calandra ${ }^{\mathrm{a}}$, D. Mompel $^{\mathrm{a}}$, V. Karalazos ${ }^{\mathrm{b}}$, S. Kaushik ${ }^{\mathrm{a}}$, M.S. Izquierdo ${ }^{\mathrm{a}}$

${ }^{a}$ Grupo de Investigación en Acuicultura (GIA), IU-ECOAQUA, Universidad de Las Palmas de Gran Canaria, Crta. Taliarte s/n, 35214 Telde, Spain

${ }^{\mathrm{b}}$ Biomar, North Shore Road, Grangemouth FK3 8UL, Scotland, UK

The authors regret.

ORIGINAL TABLE:

Table 3

Growth performance, feed utilization and somatic indexes of European sea bass (Dicentrarchus labrax) juveniles fed diets with several FM/FO contents for 90 days.

\begin{tabular}{|c|c|c|c|c|c|c|c|}
\hline & & \multicolumn{6}{|c|}{ Diets (\%FM/\%FO) } \\
\hline Initial & Standard length $(\mathrm{cm})$ & $9.1 \pm 0.1$ & $9.1 \pm 0.1$ & $9.1 \pm 0.0$ & $9.1 \pm 0.1$ & $9.1 \pm 0.0$ & $9.1 \pm 0.1$ \\
\hline \multirow[t]{5}{*}{45 days } & Feed intake $0-45(\mathrm{~kg})$ & $1.78 \pm 0.06^{\mathrm{a}}$ & $1.65 \pm 0.08^{\mathrm{abc}}$ & $1.73 \pm 0.05^{\mathrm{ab}}$ & $1.61 \pm 0.01^{\mathrm{bc}}$ & $1.65 \pm 0.07^{\mathrm{bc}}$ & $1.63 \pm 0.06^{\mathrm{bc}}$ \\
\hline & $\mathrm{g}$ feed/kg BW/day & $14.48 \pm 0.09$ & $14.08 \pm 0.55$ & $14.90 \pm 0.91$ & $14.36 \pm 0.31$ & $14.39 \pm 0.19$ & $14.34 \pm 0.25$ \\
\hline & Standard length $(\mathrm{cm})$ & $12.5 \pm 0.2^{\mathrm{a}}$ & $12.3 \pm 0.1^{\mathrm{a}}$ & $12.2 \pm 0.2^{\mathrm{a}}$ & $12.1 \pm 0.1^{\mathrm{a}}$ & $12.3 \pm 0.1^{\mathrm{a}}$ & $12.3 \pm 0.2^{\mathrm{a}}$ \\
\hline & Body weight (g) & $30.4 \pm 1.3^{\mathrm{a}}$ & $29.0 \pm 0.6^{\mathrm{ab}}$ & $28.8 \pm 1.1^{\mathrm{ab}}$ & $27.6 \pm 0.4^{\mathrm{b}}$ & $28.3 \pm 0.5^{\mathrm{ab}}$ & $28.0 \pm 1.4^{\mathrm{ab}}$ \\
\hline & $\mathrm{FE}^{3}$ & $1.04 \pm 0.02^{\mathrm{a}}$ & $1.05 \pm 0.05^{\mathrm{a}}$ & $0.98 \pm 0.07^{\mathrm{ab}}$ & $1.00 \pm 0.02^{\mathrm{ab}}$ & $1.01 \pm 0.03^{\mathrm{ab}}$ & $1.00 \pm 0.04^{\mathrm{ab}}$ \\
\hline \multirow{8}{*}{90 days } & Feed intake 0-90 (kg) & $4.90 \pm 0.10^{\mathrm{a}}$ & $4.58 \pm 0.07^{\mathrm{b}}$ & $4.61 \pm 0.05^{\mathrm{ab}}$ & $4.33 \pm 0.15^{\mathrm{bc}}$ & $4.36 \pm 0.05^{\mathrm{bc}}$ & $4.15 \pm 0.19^{c}$ \\
\hline & $\mathrm{g}$ feed $/ \mathrm{kg} \mathrm{BW} /$ day & $10.29 \pm 0.37$ & $9.97 \pm 0.37$ & $10.43 \pm 0.15$ & $10.24 \pm 0.29$ & $10.28 \pm 0.20$ & $10.34 \pm 0.05$ \\
\hline & Standard length $(\mathrm{cm})$ & $15.7 \pm 0.3^{\mathrm{a}}$ & $15.4 \pm 0.3^{\mathrm{a}}$ & $15.2 \pm 0.1^{\mathrm{ab}}$ & $15.1 \pm 0.3^{\mathrm{ab}}$ & $15.1 \pm 0.1^{\mathrm{ab}}$ & $15.0 \pm 0.3^{\mathrm{ab}}$ \\
\hline & Body weight (g) & $58.9 \pm 3.1^{\mathrm{a}}$ & $56.7 \pm 2.8^{\mathrm{a}}$ & $54.6 \pm 0.9^{\mathrm{ab}}$ & $52.2 \pm 3.3^{\mathrm{abc}}$ & $52.4 \pm 1.0^{\mathrm{abc}}$ & $49.6 \pm 2.1^{\mathrm{bc}}$ \\
\hline & Condition factor (K) & $1.5 \pm 0.1$ & $1.5 \pm 0.1^{\mathrm{a}}$ & $1.5 \pm 0.1^{\mathrm{a}}$ & $1.5 \pm 0.1^{\mathrm{a}}$ & $1.5 \pm 0.1^{\mathrm{ab}}$ & $1.5 \pm 0.1^{\mathrm{abc}}$ \\
\hline & $\mathrm{DGI}^{2} 0-90$ (\% day) & $18.2 \pm 1.1^{\mathrm{a}}$ & $17.4 \pm 1.0^{\mathrm{a}}$ & $16.6 \pm 0.3^{\mathrm{ab}}$ & $15.7 \pm 1.3^{\mathrm{abc}}$ & $15.8 \pm 0.4^{\mathrm{abc}}$ & $14.7 \pm 0.8^{\mathrm{bc}}$ \\
\hline & & \multicolumn{3}{|c|}{ Diets (\%FM/\%FO) } & \multicolumn{3}{|c|}{ Two-way ANOVA } \\
\hline & & $5 / 3$ & $0 / 0$ & $0 / 0^{+}$ & FM & FO & $\mathrm{FM} * \mathrm{FO}$ \\
\hline \multirow[t]{2}{*}{ Initial } & Standard length $(\mathrm{cm})$ & $9.1 \pm 0.1$ & $9.1 \pm 0.1$ & $9.1 \pm 0.0$ & NS & NS & NS \\
\hline & Body weight (g) & $9.8 \pm 0.3$ & $9.8 \pm 0.1$ & $9.7 \pm 0.2$ & NS & NS & NS \\
\hline
\end{tabular}

DOI of original article: https://doi.org/10.1016/j.aquaculture.2017.03.031

* Corresponding author.

E-mail address: silvia.torrecillas@giaqua.org (S. Torrecillas). 


\begin{tabular}{|c|c|c|}
\hline \multirow[t]{7}{*}{45 days } & Feed intake $0-45(\mathrm{~kg})$ & $1.58 \pm 0.03^{c}$ \\
\hline & $\mathrm{g}$ feed $/ \mathrm{kg}$ BW/day & $14.37 \pm 0.31$ \\
\hline & Standard length $(\mathrm{cm})$ & $12.1 \pm 0.1^{\mathrm{a}}$ \\
\hline & Body weight $(\mathrm{g})$ & $27.1 \pm 0.9^{b}$ \\
\hline & Condition factor $(\mathrm{K})^{1}$ & $1.5 \pm 0.1^{\mathrm{b}}$ \\
\hline & DGI $^{2}$ 0-45 (\% day) & $12.8 \pm 0.5^{\mathrm{b}}$ \\
\hline & $\mathrm{FE}^{3}$ & $0.99 \pm 0.02^{\mathrm{ab}}$ \\
\hline \multirow[t]{11}{*}{90 days } & Feed intake $0-90(\mathrm{~kg})$ & $4.09 \pm 0.11^{\mathrm{c}}$ \\
\hline & $\mathrm{g}$ feed/kg BW/day & $10.69 \pm 0.69$ \\
\hline & Standard length $(\mathrm{cm})$ & $14.7 \pm 0.3^{\mathrm{bc}}$ \\
\hline & Body weight (g) & $47.4 \pm 4.1^{\mathrm{cd}}$ \\
\hline & Condition factor (K) & $1.5 \pm 0.1^{\mathrm{abc}}$ \\
\hline & $\mathrm{DGI}^{2}$ 0-90 (\% day) & $13.9 \pm 1.4^{\mathrm{c}}$ \\
\hline & $\mathrm{FE}^{3}$ & $0.78 \pm 0.04^{b c}$ \\
\hline & $\mathrm{HSI}^{4}$ & $2.8 \pm 0.3^{\mathrm{ab}}$ \\
\hline & $\mathrm{PFI}^{5}$ & $7.4 \pm 0.8^{\mathrm{cd}}$ \\
\hline & $\mathrm{VSI}^{6}$ & $16.4 \pm 1.3^{\mathrm{ab}}$ \\
\hline & Survival (\%) & $95.6 \pm 4.0^{\mathrm{ab}}$ \\
\hline
\end{tabular}

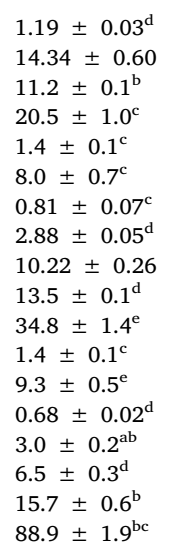

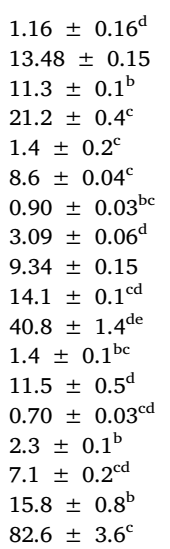

$\mathrm{P}=0.001$
$\mathrm{NS}$
$\mathrm{NS}$
$\mathrm{P}=0.001$
$\mathrm{P}=0.001$
$\mathrm{P}=0.007$
$\mathrm{NS}$
$\mathrm{P}=0.001$
$\mathrm{NS}$
$\mathrm{P}=0.012$
$\mathrm{P}=0.001$
$\mathrm{P}=0.007$
$\mathrm{P}=0.001$
$\mathrm{P}=0.01$
$\mathrm{NS}$
$\mathrm{P}=0.019$
$\mathrm{P}=0.043$
$\mathrm{NS}$

NS
NS
NS
NS
NS
NS
NS
NS
NS
NS
NS
NS
NS
NS
NS
NS
NS
NS

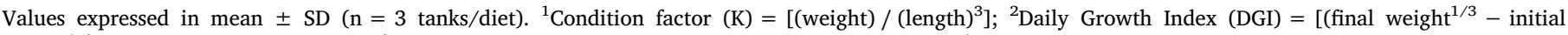

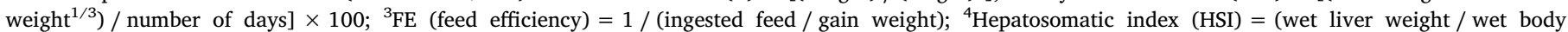

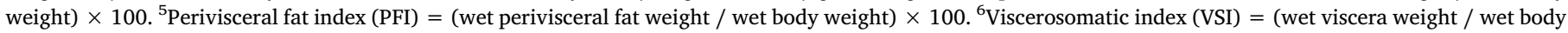

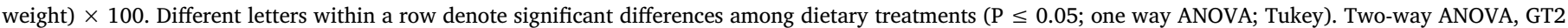

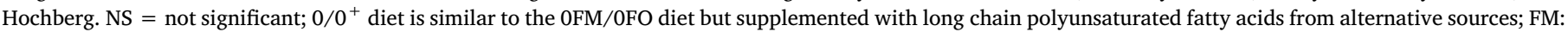
Fishmeal; FO: Fish oil.

\section{TABLE WITH CORRECT DGI:}

Table 3

Growth performance, feed utilization and somatic indexes of European sea bass (Dicentrarchus labrax) juveniles fed diets with several FM/FO contents for 90 days.

\begin{tabular}{|c|c|c|c|c|c|c|c|}
\hline & & \multicolumn{6}{|l|}{ Diets (\%FM/\%FO) } \\
\hline & & $58 / 15$ & 20-Jun & 20-Mar & 10-Jun & 10-Mar & 05-Jun \\
\hline \multirow[t]{2}{*}{ Initial } & Standard length $(\mathrm{cm})$ & $9.1 \pm 0.1$ & $9.1 \pm 0.1$ & $9.1 \pm 0.0$ & $9.1 \pm 0.1$ & $9.1 \pm 0.0$ & $9.1 \pm 0.1$ \\
\hline & Body weight (g) & $9.8 \pm 0.2$ & $9.8 \pm 0.2$ & $9.9 \pm 0.1$ & $9.9 \pm 0.3$ & $9.7 \pm 0.2$ & $9.8 \pm 0.1$ \\
\hline \multirow[t]{7}{*}{45 days } & Feed intake $0-45(\mathrm{~kg})$ & $1.78 \pm 0.06^{\mathrm{a}}$ & $1.65 \pm 0.08^{\mathrm{abc}}$ & $1.73 \pm 0.05^{\mathrm{ab}}$ & $1.61 \pm 0.01^{\mathrm{bc}}$ & $1.65 \pm 0.07^{\mathrm{bc}}$ & $1.63 \pm 0.06^{\mathrm{bc}}$ \\
\hline & $\mathrm{g}$ feed/kg BW/day & $14.48 \pm 0.09$ & $14.08 \pm 0.55$ & $14.90 \pm 0.91$ & $14.36 \pm 0.31$ & $14.39 \pm 0.19$ & $14.34 \pm 0.25$ \\
\hline & Standard length $(\mathrm{cm})$ & $12.5 \pm 0.2^{\mathrm{a}}$ & $12.3 \pm 0.1^{\mathrm{a}}$ & $12.2 \pm 0.2^{\mathrm{a}}$ & $12.1 \pm 0.1^{\mathrm{a}}$ & $12.3 \pm 0.1^{\mathrm{a}}$ & $12.3 \pm 0.2^{\mathrm{a}}$ \\
\hline & Body weight (g) & $30.4 \pm 1.3^{\mathrm{a}}$ & $29.0 \pm 0.6^{\mathrm{ab}}$ & $28.8 \pm 1.1^{\mathrm{ab}}$ & $27.6 \pm 0.4^{\mathrm{b}}$ & $28.3 \pm 0.5^{\mathrm{ab}}$ & $28.0 \pm 1.4^{\mathrm{ab}}$ \\
\hline & Condition factor $(\mathrm{K})^{1}$ & $1.6 \pm 0.1^{\mathrm{a}}$ & $1.5 \pm 0.1^{\mathrm{ab}}$ & $1.6 \pm 0.1^{\mathrm{a}}$ & $1.5 \pm 0.1^{\mathrm{ab}}$ & $1.5 \pm 0.1^{\mathrm{ab}}$ & $1.5 \pm 0.1^{\mathrm{b}}$ \\
\hline & $\mathrm{DGI}^{2} 0-45$ (\% day) & $2.1 \pm 0.1^{\mathrm{a}}$ & $2.0 \pm 0.0^{\mathrm{ab}}$ & $2.0 \pm 0.1^{\mathrm{ab}}$ & $1.9 \pm 0.0^{\mathrm{b}}$ & $2.0 \pm 0.1^{\mathrm{ab}}$ & $1.9 \pm 0.1^{\mathrm{b}}$ \\
\hline & $\mathrm{FE}^{3}$ & $1.04 \pm 0.02^{\mathrm{a}}$ & $1.05 \pm 0.05^{\mathrm{a}}$ & $0.98 \pm 0.07^{\mathrm{ab}}$ & $1.00 \pm 0.02^{\mathrm{ab}}$ & $1.01 \pm 0.03^{\mathrm{ab}}$ & $1.00 \pm 0.04^{\mathrm{ab}}$ \\
\hline \multirow[t]{13}{*}{90 days } & Feed intake 0-90 (kg) & $4.90 \pm 0.10^{\mathrm{a}}$ & $4.58 \pm 0.07^{b}$ & $4.61 \pm 0.05^{\mathrm{ab}}$ & $4.33 \pm 0.15^{\mathrm{bc}}$ & $4.36 \pm 0.05^{\mathrm{bc}}$ & $4.15 \pm 0.19^{c}$ \\
\hline & $\mathrm{g}$ feed/kg BW/day & $10.29 \pm 0.37$ & $9.97 \pm 0.37$ & $10.43 \pm 0.15$ & $10.24 \pm 0.29$ & $10.28 \pm 0.20$ & $10.34 \pm 0.05$ \\
\hline & Standard length $(\mathrm{cm})$ & $15.7 \pm 0.3^{\mathrm{a}}$ & $15.4 \pm 0.3^{\mathrm{a}}$ & $15.2 \pm 0.1^{\mathrm{ab}}$ & $15.1 \pm 0.3^{\mathrm{ab}}$ & $15.1 \pm 0.1^{\mathrm{ab}}$ & $15.0 \pm 0.3^{\mathrm{ab}}$ \\
\hline & Body weight $(\mathrm{g})$ & $58.9 \pm 3.1^{\mathrm{a}}$ & $56.7 \pm 2.8^{\mathrm{a}}$ & $54.6 \pm 0.9^{\mathrm{ab}}$ & $52.2 \pm 3.3^{\mathrm{abc}}$ & $52.4 \pm 1.0^{\mathrm{abc}}$ & $49.6 \pm 2.1^{\mathrm{bc}}$ \\
\hline & Condition factor (K) & $1.5 \pm 0.1$ & $1.5 \pm 0.1^{\mathrm{a}}$ & $1.5 \pm 0.1^{\mathrm{a}}$ & $1.5 \pm 0.1^{\mathrm{a}}$ & $1.5 \pm 0.1^{\mathrm{ab}}$ & $1.5 \pm 0.1^{\mathrm{abc}}$ \\
\hline & $\mathrm{DGI}^{2}$ 0-90 (\% day) & $1.9 \pm 0.1^{\mathrm{a}}$ & $1.9 \pm 0.1^{\mathrm{ab}}$ & $1.8 \pm 0.0^{\mathrm{ab}}$ & $1.7 \pm 0.1^{\mathrm{abc}}$ & $1.7 \pm 0.0^{\mathrm{abc}}$ & $1.7 \pm 0.1^{\mathrm{bc}}$ \\
\hline & $\mathrm{FE}^{3}$ & $0.89 \pm 0.03^{\mathrm{a}}$ & $0.91 \pm 0.03^{\mathrm{a}}$ & $0.83 \pm 0.02^{\mathrm{ab}}$ & $0.84 \pm 0.04^{\mathrm{ab}}$ & $0.84 \pm 0.02^{\mathrm{ab}}$ & $0.83 \pm 0.02^{\mathrm{ab}}$ \\
\hline & $\mathrm{HSI}^{4}$ & $2.8 \pm 0.0^{\mathrm{a}}$ & $2.5 \pm 0.1^{\mathrm{ab}}$ & $2.8 \pm 0.3^{\mathrm{ab}}$ & $2.5 \pm 0.1^{\mathrm{ab}}$ & $2.7 \pm 0.1^{\mathrm{ab}}$ & $2.5 \pm 0.0^{\mathrm{b}}$ \\
\hline & $\mathrm{PFI}^{5}$ & $8.8 \pm 0.2^{\mathrm{ab}}$ & $7.5 \pm 0.7^{\mathrm{bcd}}$ & $9.1 \pm 0.6^{\mathrm{a}}$ & $7.5 \pm 0.4^{\mathrm{bcd}}$ & $8.1 \pm 0.4^{\mathrm{abc}}$ & $7.3 \pm 0.5^{\mathrm{cd}}$ \\
\hline & $\mathrm{VSI}^{6}$ & $17.1 \pm 0.8^{\mathrm{ab}}$ & $16.0 \pm 0.4^{\mathrm{b}}$ & $18.4 \pm 1.2^{\mathrm{a}}$ & $15.9 \pm 0.1^{\mathrm{b}}$ & $17.0 \pm 0.5^{\mathrm{ab}}$ & $15.5 \pm 0.5^{\mathrm{b}}$ \\
\hline & Survival (\%) & $99.3 \pm 1.3^{\mathrm{a}}$ & $98.5 \pm 1.7^{\mathrm{a}}$ & $95.6 \pm 2.9^{\mathrm{ab}}$ & $96.3 \pm 0.6^{\mathrm{ab}}$ & $96.3 \pm 0.6^{\mathrm{ab}}$ & $96.7 \pm 1.1^{\mathrm{ab}}$ \\
\hline & & \multicolumn{3}{|c|}{ Diets $(\% \mathrm{FM} / \% \mathrm{FO})$} & \multicolumn{3}{|c|}{ Two-way ANOVA } \\
\hline & & 05-Mar & $0 / 0$ & $0 / 0^{+}$ & FM & FO & $\mathrm{FM} * \mathrm{FO}$ \\
\hline \multirow[t]{2}{*}{ Initial } & Standard length (cm) & $9.1 \pm 0.1$ & $9.1 \pm 0.1$ & $9.1 \pm 0.0$ & NS & NS & NS \\
\hline & Body weight (g) & $9.8 \pm 0.3$ & $9.8 \pm 0.1$ & $9.7 \pm 0.2$ & NS & NS & NS \\
\hline \multirow[t]{7}{*}{45 days } & Feed intake $0-45(\mathrm{~kg})$ & $1.58 \pm 0.03^{c}$ & $1.19 \pm 0.03^{\mathrm{d}}$ & $1.16 \pm 0.16^{\mathrm{d}}$ & $\mathrm{P}=0.001$ & NS & NS \\
\hline & $\mathrm{g}$ feed/kg BW/day & $14.37 \pm 0.31$ & $14.34 \pm 0.60$ & $13.48 \pm 0.15$ & NS & NS & NS \\
\hline & Standard length $(\mathrm{cm})$ & $12.1 \pm 0.1^{\mathrm{a}}$ & $11.2 \pm 0.1^{\mathrm{b}}$ & $11.3 \pm 0.1^{\mathrm{b}}$ & NS & NS & NS \\
\hline & Body weight (g) & $27.1 \pm 0.9^{b}$ & $20.5 \pm 1.0^{c}$ & $21.2 \pm 0.4^{\mathrm{c}}$ & $P=0.001$ & NS & NS \\
\hline & Condition factor $(\mathrm{K})^{1}$ & $1.5 \pm 0.1^{\mathrm{b}}$ & $1.4 \pm 0.1^{\mathrm{c}}$ & $1.4 \pm 0.2^{\mathrm{c}}$ & $\mathrm{P}=0.001$ & NS & NS \\
\hline & DGI $^{2}$ 0-45 (\% day) & $1.9 \pm 0.0^{\mathrm{b}}$ & $1.3 \pm 0.1^{\mathrm{c}}$ & $1.4 \pm 0.1^{\mathrm{c}}$ & $\mathrm{P}=0.033$ & NS & NS \\
\hline & $\mathrm{FE}^{3}$ & $0.99 \pm 0.02^{\mathrm{ab}}$ & $0.81 \pm 0.07^{c}$ & $0.90 \pm 0.03^{\mathrm{bc}}$ & NS & $P=0.05$ & NS \\
\hline \multirow[t]{9}{*}{90 days } & Feed intake $0-90(\mathrm{~kg})$ & $4.09 \pm 0.11^{\mathrm{c}}$ & $2.88 \pm 0.05^{\mathrm{d}}$ & $3.09 \pm 0.06^{\mathrm{d}}$ & $\mathrm{P}=0.001$ & NS & NS \\
\hline & $\mathrm{g}$ feed/kg BW/day & $10.69 \pm 0.69$ & $10.22 \pm 0.26$ & $9.34 \pm 0.15$ & NS & NS & NS \\
\hline & Standard length $(\mathrm{cm})$ & $14.7 \pm 0.3^{\mathrm{bc}}$ & $13.5 \pm 0.1^{\mathrm{d}}$ & $14.1 \pm 0.1^{\mathrm{cd}}$ & $\mathrm{P}=0.012$ & $\mathrm{P}=0.006$ & NS \\
\hline & Body weight (g) & $47.4 \pm 4.1^{\mathrm{cd}}$ & $34.8 \pm 1.4^{\mathrm{e}}$ & $40.8 \pm 1.4^{\mathrm{de}}$ & $\mathrm{P}=0.001$ & $\mathrm{P}=0.019$ & NS \\
\hline & Condition factor (K) & $1.5 \pm 0.1^{\mathrm{abc}}$ & $1.4 \pm 0.1^{\mathrm{c}}$ & $1.4 \pm 0.1^{\mathrm{bc}}$ & $\mathrm{P}=0.007$ & NS & NS \\
\hline & DGI $^{2}$ 0-90 (\% day) & $1.6 \pm 0.1^{\mathrm{c}}$ & $1.4 \pm 0.0^{\mathrm{e}}$ & $1.2 \pm 0.0^{\mathrm{d}}$ & $\mathrm{P}=0.001$ & NS & NS \\
\hline & $\mathrm{FE}^{3}$ & $0.78 \pm 0.04^{\mathrm{bc}}$ & $0.68 \pm 0.02^{\mathrm{d}}$ & $0.70 \pm 0.03^{\mathrm{cd}}$ & $\mathrm{P}=0.01$ & $P=0.02$ & NS \\
\hline & $\mathrm{HSI}^{4}$ & $2.8 \pm 0.3^{\mathrm{ab}}$ & $3.0 \pm 0.2^{\mathrm{ab}}$ & $2.3 \pm 0.1^{\mathrm{b}}$ & NS & $\mathrm{P}=0.001$ & NS \\
\hline & $\mathrm{PFI}^{5}$ & $7.4 \pm 0.8^{\mathrm{cd}}$ & $6.5 \pm 0.3^{\mathrm{d}}$ & $7.1 \pm 0.2^{\mathrm{cd}}$ & $P=0.019$ & $\mathrm{P}=0.011$ & NS \\
\hline
\end{tabular}




$\begin{array}{llllll}\text { VSI }^{6} & 16.4 \pm 1.3^{\mathrm{ab}} & 15.7 \pm 0.6^{\mathrm{b}} & 15.8 \pm 0.8^{\mathrm{b}} & \mathrm{P}=0.043 & \mathrm{P}=0.003 \\ \text { Survival (\%) } & 95.6 \pm 4.0^{\mathrm{ab}} & 88.9 \pm 1.9^{\mathrm{bc}} & 82.6 \pm 3.6^{\mathrm{c}} & \text { NS } & \text { NS } \\ & & & \text { NS }\end{array}$

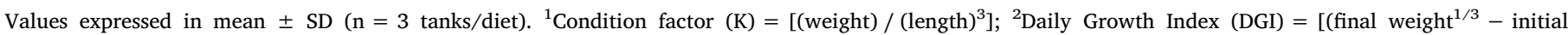

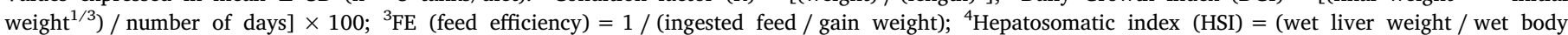

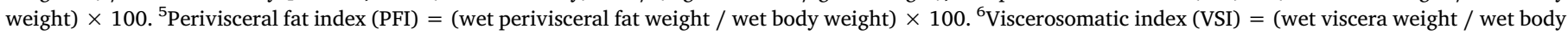

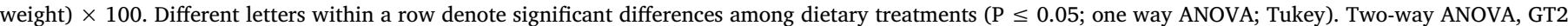

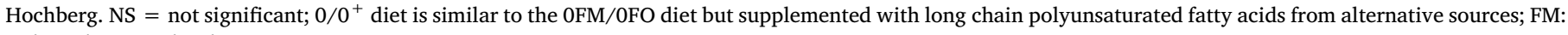
Fishmeal; FO: Fish oil.

The authors would like to apologise for any inconvenience caused. 